(Aus der bakteriologisch-serologischen Abteilung des Allgemeinen Krankenhauses St. Georg. [Leiter: Priv.-Doz. Dr. E. Jacobsthal].)

\title{
Uber Phagocytoseversuche mit Myeloblasten, Myelocyten und eosinophilen Leukocyten (mit Bemerkungen uiber den feineren Bau der eosinophilen Lenkocyten).
}

\author{
Ton \\ E. Jacobsthal. \\ Mit 5 Textabbildungen. \\ (Eingegangen am 2\%. April 1921.)
}

Úber die Fähigkeit der neutrophilen Leukocyten zur Phagocytose und über die Morphologie und Biologie dieses Vorganges gibt es zahlreiche Untersuchungen. In neuerer Zeit ist auch die Frage der Phagocytose durch Lymphocyten, insbesondere in den Arbeiten von Bergel näher erforscht worden; Bergel hat gezeigt, daß es namentlich die Fette und fettartigen Substanzen sind, die die Tätigkeit dieser Zellart anregen. Morkwürdigerweise aber liegen über die Freßtätigkeit der Mutterzellen der Leukocyten, nämlich der Myeloblasten, Myelocyten und der sogenannten Übergangsformen, sowie der selteneren normalen Z llen des Blutes, wie der Eosinophilen und Mastzellen, soweit mir bekannt ist, keine Untersuchungen vor. Vorliegende Arbeit soll ein Baustein zur Ausfüllung dieser Lücke sein ${ }^{1}$ ) .

Die von mir getroffene Versuchsanordnung ist äußerst einfach und entspricht der bei sonstigen Phagocytoseversuchen üblichen.

Es wurde aus der Vene des Patienten Blut entnommen und sofort mit Natr.-citr.-Lösung 5 proz. so gemiseht, daß die Natriumcitriumlösung 1 pr z. wurde Es wurde im Eisschrank absetzen gelassen und nach 1-3 Stunden wurde die milchige Leukocytenschicht mit Glascapillaren entnommen. Bei den Versuchen wurde diese Leukocytenemulsion zu gleichen Teilen mit der Aufschwemmung der zu phagocytierenden Substanz versetzt und die Mischung bei $37^{\circ}$ bebrütet.

Zur Untersuchung diente mir das Blut von folgenden Fällen: 1. Akute Myeloblastenleukämie Fall Behn. Dieser Fall ist

1) Durch Hexrn O. Schilling-Torgau wurde ich darauf aufmerksam gemacht, daß L. Nattan-Larvier et Parvu (C. r. soc. biol. 1909, p. 574) bereits Phagocytenversuche mit den Eosinophilen aus dem Blute von Filariakranken gemacht haben. 
von Arning, Paschen und mir seinerzeit schon besprochen worden (Hamburger Ärzte-Corresp. 1916, Nr. 6, S. 60). Fs handelte sich um ein 14-15jähriges Mädchen, das akut fieberhaft mit Blutungen an Haut und Zahnfleisch und mit Schmerzhaftigkeit der Knochen erkrankt war. Es traten dann gangränöse Prozesse an der Mundschleimhaut auf. Keine Milz- und Lymphdrüsenschwellung; zunehmender Verfall. Das Blut zeigt das Bild der akuten Myeloblastenleukämie Naegelis; Blutbild beherrscht von großen einkernigen Myeloblasten mit eigenartigen Vakuolen. Fehlen der Eosinophilen, Mastzellen und Blutplättchen; reichliche Normoblasten.

Das Blutbild entspricht am meisten dem in $\mathrm{Pappenheims} \mathrm{Atlas}$ (Supplementband Tafel 33, Prototyp 56) abgebildeten Fall. Ganz auffallend sind die großen Vakuolen der Myeloblasten. Ich selbst habe nachgewiesen, daß die Substanz dieser Vakuolen auf keinen Fall Fett sein kann (Osmium- und Sudanfärbungsversuche). Ich fand im ungefärbten, angetrockneten Präparat bei Beobachtung mit Immersion die „Vakuolen“ als einzige Details an dem sonst weißen Körper der Myeloblasten sich als rötlich-gelbe, kuglige Gebilde hervorheben. Olsen hat geglaubt, die Blutplättchenfreiheit mit den Vakuolen in Verbindung bringen zu sollen und hat die Vakuolen als das Produkt phagocytierter Blutplättchen aufgefaßt.

2. Myeloische Leukämie. Fall Mert. Etwa 52jähriger Mann, Diagnose auf myeloische Leukämie vor etwa 4 Jahren gestellt. Damals starker Milztumor. Die Zahl der weißen Blutkörperchen betrug über 260000 , vorwiegend waren es Myelocyten der verschiedensten Reifegrade, vor allem auch sehr viele unreife eosinophil und basophil gekörnte Myelocyten. Der Fall war dadurch so besonders bemerkenswert, daß durch höchst intensive Röntgenbestrahlung im Röntgeninstitut unseres Krankenhauses (Prof. Albers-Schönberg) es wiederholt gelang, die Zahlen der weißen Blutkörperchen auf die Norm oder nur mäßig erhöhte Grade zurückzuschrauben; während sich in den bisher beschriebenen Fällen und Dosierungen Rezidive kaum beeinflussen ließen. Doch soll darauf nicht näher eingegangen werden. Uns interessiert hier nur, daf dieser Fall ein sehr bequemes Versuchsobjekt war, bei dem sich ohne Mühe eine milchige Leukocytenemulsion gewinnen ließ. Anführen möchte ich hier noch, daß ich bei diesem Fall einmal 18 Stunden nach einer intensiven Röntgenbestrahlung Blut entnommen und die Emulsion der weißen Blutzellen bebrütet habe. Hierbei entstanden aus den Leukocyten und Myelocyten, wahrscheinlich durch die vorhergehende Röntgenschädigung ganz eigenartige, bisher noch nicht beschriebene Gebilde: Die Zellen bildeten breite Auslänfer und zwar 1-3, in der Peripherie dieser Ausläufer findet man Kernreste die offenbar von den pyknotischen Kernen stammen und selbst pyknotisch sind. Die Beschreibung der Einzelheiten und experimentelle Bearbeitung der Fragen behalte ich mir vor.

3. Hodgkinsche Krankheit mit multiplen Infiltraten der Haut und hochgradiger Eosinophilie.

Dieser höchst eigenartige Fall ist kliniseh keineswegs geklärt. Er wird noch, wie mir Professor Paul Lazarus freundlichst mitteilte, in seinem klinisehen Verhalten und besonders wegen seiner günstigen Beeinflussung durch Radium- 
bestrahlung genauer beschrieben werden. Es handelte sich um ein etwa 45 jähriges Fräulein Pri., J.-Nr. E., S. 603, das am stärksten am Kinn, an Unterarmen und Brust hochgradige tumorartige Hautinfiltrate aufwies. Drüsenschwellungen mittleren Grades in der oberen Körperhälfte. Uns interessiert hier nur das Blutbild. Es bestand nämlich eine so hochgradige Eosinophilie, wie man sie nur selten findet, nämlich gegen $50 \%$, neben $21 \%$ Leukocyten und $25 \%$ Lymphocyten. In den Zellemulsionen mit Natriumcitrat fanden sich vielleicht durch das Verkleben der Leukocyten miteinander gegen $80 \%$ eosinophile Zellen, die auch im ungefärbten Präparat durch ihre Granula leicht kenntlich waren ${ }^{1}$ ).

Als Versuchsobjekt benutzte ich für Fall 1 eine dünne Zinnoberaufschwemmung, für Fall 2 und 3 Aufschwemmungen von Zinnober, Milzbrandbacillen, Mikrococcus tetragenus (Sarcina tetragina), Schildkrötentuberkelbacillen, menschlichen Tuberkelbacillen, Hammelblutkörperchen und Staphylokokken. Es zeigte sich, daß das letztgenannte Objekt, die Staphylokokken, für solche Versuche ungeeignet sind. Sie haben nämlich die Eigenschaft, aus dem Plasma das Fibrin auszufällen, ein Verhalten, das $\mathrm{Much}$ schon vor Jahren beschrieben hat. Die Untersuchung der im Fibrinnetz gefangenen Zellen ist natürlich eine unnütze Erschwerung der Versuche. Die Milzbrandbacillen, die unter Umständen auch die Fibrinbildung fördern können, sind kein besonders günstiges Versuchsobjekt. Sie bilden nach mehreren Stunden sehr lange Fäden mit den schönsten Kapseln; aber es ist nur natürlich, daß das Zellprotoplasma, um sie in sich aufzunehmen, sich ganz gewaltig dehnen muß. Die Fäden sind dann von dem schmalen Protoplasmasaum eingehüllt, ähnlich wie man das zuweilen bei Fremdkörperriesenzellen beobachten kann, die sich an und um Baumwollfäden entwickelt haben.

Auch die Benutzung von Hammelblut erwies sich als nicht sehr zweckmäßig, weil menschliches Serum bekanntlich in wechselndem Maße die Fähigkeit hat, Hammelblut aufzulösen (normale Amboceptoren). So wird nur ein Teil der roten Blutscheibchen phagocytiert. Wollte man diesen Fehler ausschalten, so müßte man das Zellmaterial waschen, was aber auch Nachteile mit sich bringt. Als beste Versuchsobjekte kann ich Zinnober, Mikrococcus tetragenus und säurefeste Stäbchen empfehlen. Die Beobachtung geschah stets im frischen Präparat, zuweilen auch mit Dunkelfeldbeleuchtung, und im gefärbten Präparate. Die Präparate zur Färbung werden durch Ausstreichen nach den Regeln der hämatologischen Technik gewonnen. Aber selbst bei peinlichster Technik ist die Zinnoberphagocytose im gefärbten Präparate nur schwer darstellbar, offenbar weil die spitzen Zinnoberpartikel beim Ausstreichen die Zellen zerstören; im ungefärbten Präparate erhält man aber hier die prachtvollsten Bilder (s. Abb.). Umgekehrt ist die Phagocytose von

1) Dieser auffallende Befund, daß sich in der Aufschwemmung mehr Eosinophile ${ }_{\text {f }}$ anden als bei den Ausstrichpräparaten, wird vielleicht zu den Befunden Li ebreichs (Sohweizer med. Wochenschr. 1921, Nr. 12) Beziehung haben. 
Bakterien am besten im gefärbten Präparate zu erkennen. Die farbenprächtigsten Bilder erhielt ich durch Kombination der Gramfärbung mit der M a y-Grünwald-Färbung für eosinophile Zellen und panoptischer Färbung für Myelocyten usw. Chinesische Tusche fand ich für Phagocytoseversuche nicht so sehr zweckmäßig.

Ich komme nun zu dem Ergebnis meiner Versuche. Ich fand, daß die M yeloblasten eine ganz außerordentlich rege Freßtätigkeit gegen Zinnober entwickeln (s. Abb. 1). Sie ging so weit, daß die Zellen sich bis zum Platzen mit den Fremdkörpern beladen und dadurch zuweilen ganz abstruse Formen bilden können. Der Kern wird dadurch völlig an den Rand der Zelle gedrückt. Wie schon erwähnt, habe ich leider mit den

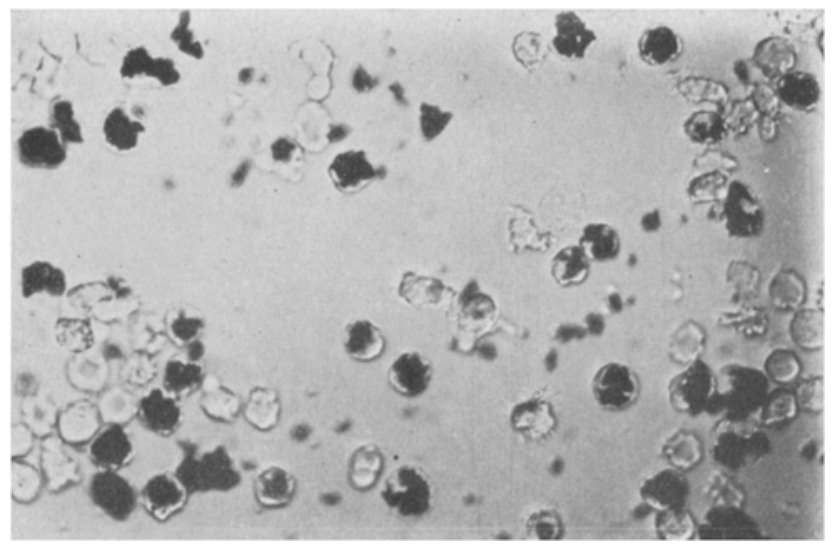

Abb. 1. Myeloblastenlenkämie (Fall Behn). Zinnoberphagocytose. Ungefärbtes Präparat.

Myeloblasten keine Versuche an anderen Objekten gemacht. Ich fand die Fähigkeit der Myeloblasten zum Phagocytieren noch nach 48-60 Stunden Eisschrankaufenthalt erhalten. Die Phagocytose durch diese Zellart geht sehr schnell vor sich, und man kann im Dunkelfeld auch sehr schön ihre langen, stacheligen amöboiden Fortsätze erkennen.

Die Versuche mit M yeloc y te n hatten ein hiervon etwas abweichendes Ergebnis bei beiden geprüften Fällen. Nach längerem Suchen konnte man zwar hin und wieder ganz vereinzelte Phagocytosen entdecken, niemals aber waren sie lebhaft oder reichlich. Das von mir beobachtete Material enthielt in größten Mengen normale und pathologische Myelocyten aller Reifegrade und mit den verschiedensten Granulationsformen; vor allem auch reichlich eosinophil und basophil granulierte Myelocyten. Auch die sogenannten Übergangsformen waren nicht lebhaft in ihrer Freßtätigkeit, während ich die Leukocyten dieser Fälle, wenn auch nicht besonders stark, so doch immerhin normal phagocytär fand (s. Abb. 2 u. 3). 


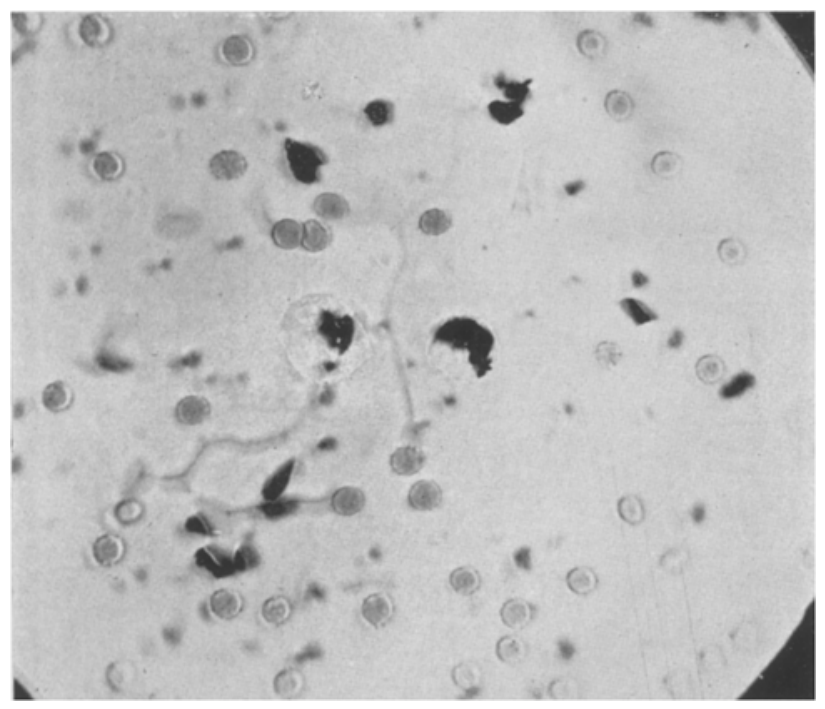

Abb. 2. Myeloische Leukämie (Fall Mert). Zimoberphagocytose beỉ zwei Myelocyten. Ungefärbtes Präparat.

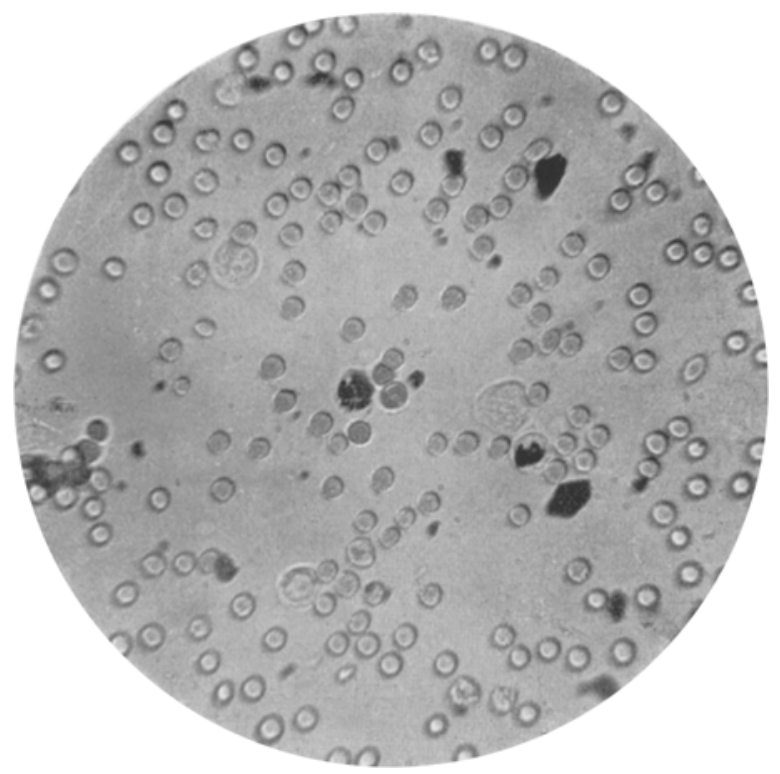

Abb. 3. Myeloische Leukämie (Fall Mert). Nur mäßige Phagocytose. Ungefärbtes Präparat. 
Die bemerkenswertesten Ergebnisse hatte ich bei den e o sin o philen Zellen des Falles III. Die Beobachtung der Eosinophilen im frischen und gefärbten Präparate ist ja ganz besonders einfach. Auch im frischen Präparat kann man sie mit keiner anderen Zellart verwechseln. Bei dieser Zellart bestehen besondere Verhältnisse dadurch, daß das Protoplasma mit den Granulis meistens ganz prall gefüllt ist; es ist nur natürlich, $\mathrm{daB}$ dadurch eine gewisse, fast möchte ich sagen, Unbeholfenheit der Zellbewegung entsteht, die erst durchbrochen, werden muß. So erklärt sich, daß so grobe Objekte wie die Zinnoberpartikelchen von den Eosinophilen fast gar nicht aufgenommen werden, ich will allerdings nicht sicher behaupten, daß das nur einen mechanischen Grund hat. Es könnten auch chemische Verhältnisse da eine Rolle spielen, ja diese Annahme gewinnt durch die Beobachtung der Tuberkelbacillenphagocytose an Wahrscheinlichkeit.

Die Eosinophilen phagocytierten Staphylokokken und Mikrococcus tetragenus ziemlich stark; aber die $\mathrm{Phagocytose} v$ on säurefesten Stäbchen warso enorm intensi $v$, daß die Zellen zu unförmlichen Haufen wurden. Nur schwer war noch im gefärbten Präparat Kern und Protoplasma zu erkennen. Ich möchte fast vermuten, daß irgendein noch unbekannter Zusammenhang zwischen den fettartigen Substanzen der Tuberkelbacillen und der eosinophilen Substanz besteht, die ja in ihren Färbeeigenschaften so viel Verwandtschaft zu den lipoidhaltigen roten Blutscheiben aufweist.

Besonders wichtig erscheinen mir die Beobachtungen, die ich über das Protoplasma gemacht habe. Wenn man die phagocytierende Tätigkeit der Eosinophilen im frischen Präparate, besonders aber auch nach 10-18stündigem Aufenthalte im Brutschrank beobachtet, so sieht man, daß diese unbeholfenen Zellen ganz lange schmale, oft geradezu hirschgeweihartige Fortsätze, zuweilen sogar nach mehreren entgegengesetzten Richtungen, ausgesandt haben. Diese Beweglichkeit und $d a ß$ es sich nicht etwa um Leichenersicheinungen, sondern um aktive Beweglichkeit handelt, erkennt man bei längerer Beobachtung ein und derselben Zelle - kann die Zelle nur mit einem gewissen Opfer erreichen. Dieses Opfer ist ein Teil ihrer eosinophilen Granula. Bei Betrachtung solcher verzweigten Zellen sieht man nun, daß das Protoplasma der Fortsätze ganz oder fast ganz homogen erscheint, und nur an der einen oder anderen Stelle dieser Fortsätze an der Mitte oder an ihrem Ende sieht man noch einzelne eosinophile Granula, zuweilen in molekularer Bewegung ${ }^{1}$ ). Suchen wir nun nach Ửbergängen zwischen der ruhenden und der so lebhaft bewegten eosinophilen Zelle, so finden wir einzełne

${ }^{1)}$ Auch kann man am frischen Präparat verfolgen, wie sich einzelne eosinophile Granula aus dem Verbande lösen und unter molekularer Bewegung in die umgebende Flüssigkeit übergehen. 
Individuen, die in fast amöbenartiger Weise ein eosinophil granuliertes Entoplasma und ein homogen erscheinendes, hier und da vorgebuchtetes Ektoplasma besitzen. Auf der Abb. 4 sind solche Zellen im ungefärbten Präparate zu sehen. Färbt man nun die Zellen in diesem Stadium, so erkennt man zu seinem Erstaunen, daß das homogene Ektoplasma ausgesprochen basophil ist, also bei panoptischer Färbung die blaue Farbe annimmt. Sieht man genauer zu, so ergibt sich, daß das Ektoplasma vom Entoplasma nicht prinzipiell verschieden ist; denn es zeigt sich, daß die Grundsubstanz des vorher als Entoplasma bezeichneten Teiles ebenfalls basophil ist und sich vom Ektoplasma nur durch die Einlagerung der eosinophilen Granula unterscheidet.

Wir kommen damit zu einer völlig ne uen A uffassung des Baues der eosinophilen Zelle. Wir haben bei ihr danach eine basophile plasmatische Grundsubstanz anzunehmen, in die die beiden

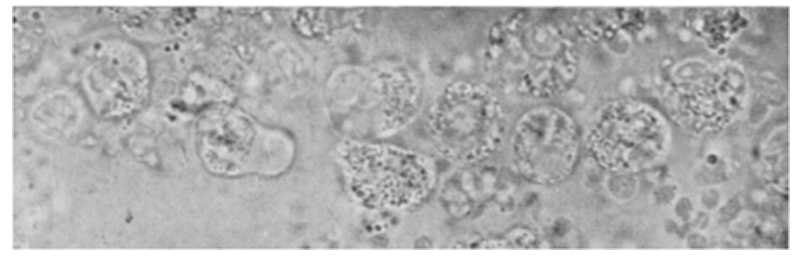

Abb. 4. Amöboide Bewegung von Eosinophilen. Ungefärbtes Präparat. Man erkennt das vorgebuchtete granulafreie Ektoplasma an zwei Zellen.

Arten von Gran ula, nämlich die eosino philen und die von Naegeli beschriebenen stark lichtbrechenden, für die ich den Namen Glasgranula vorschlagen möchte, eingeschlossen sind. Das Protoplasma hat nach Ausstoßung eosinophiler Granula die Fähigkeit lebhafter Phagocytose. Na egeli hat ähnliches offenbar schon gesehen, aber nicht ganz richtig gedeutet, indem er sagt: „Das Protoplasma zeigt nur bei Methylenblaufärbung eine schwache Andeutung des basophilen Reticulums; sonst ist es völlig erfüllt von Granulis." Diese Auffassung des Protoplasmas als Reticulum gibt ein durchaus falsches Bild, denn das Protoplasma ist, wie ich gezeigt habe, als ho mogene Grundsubstanz ein für aich bewegliches Organ. Wenn man erst diese Auffassung des Baues der eosinophilen Zelle sich zu eigen gemacht hat, gelingt es auch, in nativen, gefärbten Blutausstrichpräparaten von Fällen mit starker Eosinophilie das basophile Protoplasma in einzelnen, weniger granulareichen Zellen zu sehen.

Die beigefügte Abbildung (Abb. 4) von ungefärbten eosinophilen Zellen läßt die amöbenartige Trennung von Ektoplasma und Entoplasma einer eosinophilen Zelle deutlich erkennen. Leider sind die hirschgeweih- 
artigen Fortsätze phagocytierender Zellen nicht im lebenden Präparat aufgenommen worden.

Die Kerne der easinophilen Zellen sind, wie auch Naegeli angibt, außerordentlich charakteristisch, sie sind polymorph, aber etwas weniger als die der Neutrophilen, und etwas chromatinärmer. Es ist nun bekannt, daß man bei tuberkulösen Prozessen und auch in Asthmalungen zuweilen Zellen mit blasigen Kernen und eosinophilen Granulis findet, deren Zugehörigkeit zu den echten Eosinophilen aber wegen ihres abweichenden Kernbaues zweifelhaft gewesen ist. Ich habe nun bei meinen Untersuchungen den Eindruck gewonnen, als ob derartige, blasige, große blasse Kerne sich in solehen Eosinophilen entwickeln könnten, die nach Granulaschwund ihre phagocytäre Tätigkeit ausgeübt haben. Diese Ergebnisse, die ich vorläufig noch mit einer gewissen Zurückhaltung äußern möchte, würden die eigenartigen Befunde an den Lungen er-

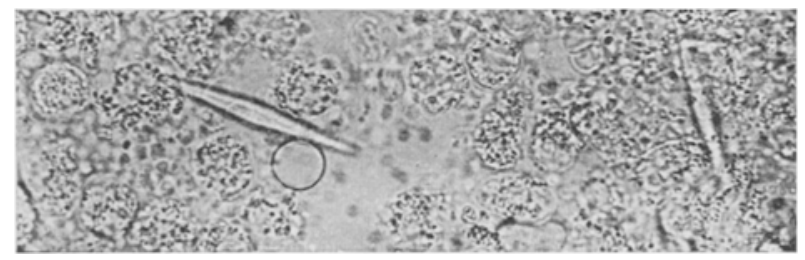

Abb. 5. Bildung von Charcot-Leydenschen Krystallen aus Eosinophilen.

klären: durch die Phagocytose und vielleicht durch die Tätigkeit als Wanderzelle ändert die eosinophile Zelle einen Teil ihrer morphologischen Eigenart.

Schließlich habe ich noch Autolyseversuche mit Zellbrei aus eosinophilen Zellen gemacht. Ich konnte beobachten, wie sich, besonders bei auch nur geringer Eintrocknung des Materials, die schönsten CharcotLe yde nschen Asthmakrystalle in großer Menge und in den verschiedensten Größen unter dem Deckglas entwickelten. Da meine Zellaufschwemmung über $80 \%$ eosinophile Zellen enthielt, und da aus Leukocyten allein die Bildung derartiger Krystalle nicht erfolgt, so sehe ich in diesen Befunden einen wichtigen Beitrag zur Entstehung solcher Krystallbildungen bei Asthma bronchiale aus Eosinophilen.

Mag auch der Zusammenhang $z$ wischen Eosinophilen und Asth makrystallen häufig angenommen worden sein, so schließt sich der Ring der Beweise durch meine direkte Beobachtung der Entstehung von Asthmakrystallen aus Eosinophilen. Ich hatte wiederholt den Eindruck, als ob die Krystallbildung direkt im Protoplasma der eosinophilen Zelle begönne. Die beigegebene Photographie (Abb. 5) des ungefärbten Materials zeigt sehr deutlich die Entstehung der rhombischen Asthmakrystalle aus der Zellemulsion. 
20 E. Jacobsthal: Über Phagocytoseversuche mit Myeloblasten usw.

Die Ergeb nis se vorliegender Untersuchungen kann ich kurz folgendermaßen zusammenfassen:

1. Die Mutterzellen der neutrophilen Leukocyten, nämlich die Myeloblasten und Myelocyten, haben die Fähigkeit, in vitro Materialien verschiedener Art, wie Zinnober und Bakterien, zu phagocytieren (Versuche an myeloischem Citratblut).

2. Der eosinophile Leukocyt besitzt ebenfalls die Fähigkeit phagocytärer Tätigkeit.

3. Durch die Beobachtung lebender und gefärbter phagocytierender eosinophiler Zellen wird es deutlich, daß diese Zellart ein basophiles homogenes Protoplasma besitzt, in das die typischen Granula, nämlich 1. die eosinophilen Granula, und 2. die stark lichtbrechenden Einschlüsse $\mathrm{Na}$ egelis, für die hier die Bezeichnung ,G G as g ran u la" vorgeschlagen wird, eingebettet sind.

4. Die phagocytäre Tätigkeit der Eosinophilen führt zu einer Trennung des granulierten Entoplasmas von der basophilen Grundsubstanz, die so als Elktoplasma imponiert. Diese Grundsubstanz kann schmale, hirschgeweihartige Fortsätze bei der Phagocytose ausstrecken.

5. In phagocytierenden, eosinophilen Zellen scheint sich ein blasiger, blasser, großer Kern entwickeln zu könnenen.

6. Es wurde die direkte Entstehung Charcot-Leydenscher Krystalle aus eosinophilen Zellen beobachtet (Beziehung zwischen Asthmakrystallen und Eosinophilen). 